\title{
Editorial
}

\section{New trends in nanophotonics}

https://doi.org/10.1515/nanoph-2020-0170

Nanophotonics considers the complex interactions between light and matter at the sub-wavelength scale. Recent progress in nanophotonics has revealed unprecedented optical phenomena, which have opened up the novel and rapidly developing fields of metamaterials, photonic crystals, and plasmonics. The last few decades have seen explosive growth in this field, from fundamental research to applications including condensed-matter physics, quantum photonics, near-field/far-field optics, biochemical sensing, deep learning for nanophotonic design, and nanofabrication/ nanomanufacturing.

The International Conference on Metamaterials, Photonic Crystals, and Plasmonics (META) is an annual conference of researchers in metamaterials, nanophotonics, and other closely related topics. It covers a broad range of topics including, but not limited to, metasurfaces, meta-devices, topological effects in optics, two-dimensional materials, light-matter interaction in nano-cavities, plasmonic circuits, thermal engineering, and quantum photonic systems. The latest conference, META'19, was held in Lisbon, Portugal (July 23-26, 2019), where the latest trends and recent progress in nanophotonics were discussed to provide further insights for researchers. This special issue introduces a selection of cutting-edge original research and review papers from the conference.

Metasurfaces can manipulate the optical properties of light with the ultrathin materials. Two groups review this topic in detail in this issue. Wei et al. [1] focus on the recent research progress in metasurfaces for holographic displays, polarization conversion, active modulation, and linear and nonlinear modulation; the authors discuss in detail the working principle and advantages of metasurfaces and provide many specific applications. Intaravanne and Chen [2] review the recent progress on metasurfaces, mainly focusing on metasurface devices for polarization detection and polarization profiles. The review also presents various applications of metasurface-based devices by manipulating arbitrary polarizations, with specific examples for high-resolution images, quick-response codes, color images, and holograms.

Other topics on metasurfaces deal with the unique metasurface-driven optical properties, presented by three groups. Mun et al. [3] report a method to modulate the phase information of a Fano-resonant metasurface; they present an optical de-multiplexer that covers a broad wavelength range in the near-infrared region, and experimentally verify the extremely small full width at half-maximum. The proposed design approach can provide complete control of Fanoresonant metasurfaces for various applications including optical multiplexers, filters, and switches. Park et al. [4] present a metasurface deflector that can control the direction of emission of colloidal quantum dots; the design of metasurfaces was adapted from the optical Yagi-Uda nanoantenna, and the deflection efficiency of the device reaches up to $70 \%$. The developed metasurface facilitates the integration of metasurfaces into a resonant nanocavity, which can lead to the rapid development of many active devices. As another metasurface device, Park et al. [5] demonstrate a general non-Hermitian metasurface to observe exceptional points that exhibit both complex eigenvalues and eigenvectors of a system to form defective eigen-spaces. The designed metasurface is composed of two orthogonally oriented split-ring resonators in a unit cell and exhibits an exceptional point in the polarization space of light. These results provide a platform to study non-Hermitian physics and sensitive terahertz systems that have nontrivial phase responses.

This issue also presents the combination of nanophotonics with deep learning, which has seen an explosion in interest recently. Specifically, the combination of deep learning and inverse design in nanophotonics is reviewed comprehensively by So et al. [6], who discuss recent progress in the application of deep learning to inverse design in nanophotonic devices, as categorized by three paradigms of the learning method. In addition, Jiang and Fan [7] present a global optimization algorithm that uses deep learning to design metasurfaces. The proposed optimization method considers populations and a generative neural network that is trained to optimize the population of the devices. The process allows efficient global optimization, which can be extended to general inverse design problems in other areas of physics. 
Various applications of photonic devices are also discussed in this issue, such as highly sensitive sensors [8, 9], a nonlinear optical device [10], a plasmonics electro-optic device [11], and a multipole engineering method [12]. Qian et al. [8] propose an inexpensive and easy-to-fabricate fiber-optic localized surface plasmon resonance (SPR) sensor to detect charge and discharge processes in supercapacitors. Because of using localized SPR, the proposed sensor is insensitive to changes in ambient temperature and therefore more practical than existing devices. Ndao et al. [9] also report on a highly sensitive biomedical sensor that exploits bound states in the continuum to differentiate exosome secretion from a single cell. The proposed sensor exhibits strong overlap between the medium supporting the mode and the analytes, and thereby achieves a high figure of merit and high sensitivity. These results may provide a platform for highly conductive optical sensors for various biomedical applications. Fujii and Tanabe [10] review the nonlinear optics of whispering-gallery-mode microresonators, focusing on dispersion engineering, calculation method, and measurement method for the generation of Kerr-frequency combs. Vinnakota et al. [11] present a semiconductor-based optoelectronic switch for functional plasmonic circuits; the active modulation of surface plasmon polaritons allows switching rates up to $1 \mathrm{~Gb} / \mathrm{s}$ and therefore shows the potential to achieve high data rates and fast optoelectronic devices. Liu et al. [12] comprehensively review the topic of multipole and multimode engineering in metasurface devices; these concepts can be applied in various nontrival intermodal couplings as well as multipolar interferences of meta-atoms and meta-molecules. The review provides comprehensive perspectives for further development of dielectric-based nanophotonics.

On the advances in nanofabrication methods, Jeong et al. [13] review recent progress on 3D and 4D printing that combine active and responsive functions to 3D printing in the field of optics and metaphotonics. They discuss designs and applications of 3D and 4D printing technologies and highlight the opportunities to explore applications in optics and metaphotonics.

Over the last few decades, nanophotonic devices have enabled realizing unprecedented optical phenomena. This issue also presents numerous noticeable discussions of novel optical phenomena. Tang et al. [14] review recent developments in flat-band localization and associated phenomena in optically induced flat-band photonic lattices. Flat-band systems have completely dispersionless bands within the whole Brillouin zone, so they allow the exploration of exotic ground-state properties of many-body systems. This review may provide a platform to study the fundamentals and applications of flat-band physics. Pacheco-Pena et al. [15] present a brief review on conformal mapping as an analytical frame for nanoantennas. Specifically, the method focuses on mainly bow-tie nanoantennas as an asymmetric design that is applied to the nonradiative Purcell enhancement of a nearby nanoemitter. By showing the advantages of conformal mapping, the paper provides physical insights into the design of various symmetric and asymmetric nanoantennas.

Finally, concerning the high demand for active and tunable photonic devices, Abdollahramezani et al. [16] review the state of the art in tunable nanophotonics enabled by chalcogenide phase-change materials. These materials can be easily integrated into nanophotonic platforms and thereby provide reconfigurability of optical functionalities. The paper discusses the unique properties of materials, structural transformation, electro-optic, and thermo-optic effects of chalcogenide phase-change materials, and then provides perspectives for further development of such materials with tunable properties.

This special issue provides novel perspectives on new trends in nanophotonics. We appreciate the efforts and contributions of all authors, and hope that this special issue will inspire other researchers in the rapidly expanding field of nanophotonics.

Acknowledgment and funding: This work was supported by the National Research Foundation (NRF) grants (NRF-2019R1A2C3003129, CAMM-2019M3A6B3030637, NRF-2019R1A5A8080290, NRF-2018M3D1A1058997) funded by the Ministry of Science and ICT (MSIT), Republic of Korea. S.S. acknowledges a global Ph.D. fellowship (NRF2017H1A2A1043322) from the NRF-MSIT, Korea.

\section{References}

[1] Wei Q, Huang L, Zentgraf T, Wang Y. Optical wavefront shaping based on functional metasurfaces. Nanophotonics 2020.

[2] Intaravanne Y, Chen X. Recent advances in optical metasurfaces for polarization detection and engineered polarization profiles. Nanophotonics 2020.

[3] Mun S-E, Choi C, Hong J, Lee B. Broadband wavelength demultiplexer using Fano-resonant metasurface. Nanophotonics 2020. 
[4] Park Y, Kim H, Lee J-Y, Ko W, Bae K, Cho K-S. Direction control of colloidal quantum dot emission using dielectric metasurfaces. Nanophotonics 2020.

[5] Park S, Lee S-G, Baek S, et al. Observation of an exceptional point in a non-Hermitian metasurface. Nanophotonics 2020.

[6] So S, Badloe T, Noh J, Bravo-Abad J, Rho J. Deep learning enabled inverse design in nanophotonics. Nanophotonics 2020.

[7] Jiang J, Fan JA. Simulator-based training of generative neural networks for the inverse design of metasurfaces. Nanophotonics 2020.

[8] Qian S, Chen X, Jiang S, et al. Direct detection of charge and discharge process in supercapacitor by fiber-optic LSPR sensors. Nanophotonics 2020.

[9] Ndao A, Hsu L-Y, Cai W, et al. Differentiating and quantifying exosomes secretion from a single cell using quasi-bound states in the continuum. Nanophotonics 2020.

[10] Fujii S, Tanabe T. Dispersion engineering and measurement of whispering gallery mode microresonator for Kerr frequency comb generation. Nanophotonics 2020.

[11] Vinnakota RK, Dong Z, Briggs AF, Bank SR, Wasserman D, Genov DA. Plasmonic electro-optic modulator based on degenerate semiconductor interfaces. Nanophotonics 2020.

[12] Liu T, Xu R, Yu P, Wang Z, Takahara J. Multipole and multimode engineering in Mie resonance-based metastructures. Nanophotonics 2020.

[13] Jeong HY, Lee E, An S-C, Lim Y, Jun YC. 3D and 4D printing for optics and metaphotonics. Nanophotonics 2020.

[14] Tang L, Song D, Xia S, et al. Photonic flat-band lattices and unconventional light localization. Nanophotonics 2020.

[15] Pacheco-Pena V, Alves RA, Navarro-Cia M. From symmetric to asymmetric bowtie nanoantennas: electrostatic conformal mapping perspective. Nanophotonics 2020.

[16] Abdollahramezani S, Hemmatyar O, Taghinejad H, et al. Tunable nanophotonics enabled by chalcogenide phase-change materials. Nanophotonics 2020.

\section{Sunae So}

Department of Mechanical Engineering, Pohang University of Science and Technology (POSTECH), Pohang 37673, Republic of Korea. https://orcid.org/0000-0001-8606-2234

\section{Namkyoo Park}

Photonic Systems Laboratory, Department of Electrical and Computer Engineering, Seoul National University, Seoul 08826, Republic of Korea. https://orcid.org/0000-0003-0197-7633

\section{Hak Joo Lee}

Center For Advanced Meta-Materials and Division of Applied Nanomechanics, Korea Institute of Machinery and Materials (KIMM), Daejon 34103, Republic of Korea. https://orcid.org/0000-0002-2573-1761

\section{Junsuk Rho}

Department of Mechanical Engineering and Department of Chemical Engineering, Pohang University of Science and Technology (POSTECH), Pohang 37673, Republic of Korea, e-mail: jsrho@postech.ac.kr. https://orcid.

org/0000-0002-2179-2890 